group the Warwick- Edinburgh Mental Well-being Scale (WEMWS) was used to measure outcomes. The mean wellbeing score pre and post programme saw an increase of 7.2, demonstrating a positive change.

Conclusions Results from the pilot group have demonstrated participants' increased knowledge and understanding of heart failure. They developed coping mechanisms, changes in behaviour and benefited from peer support. Measuring decrease in medical intervention over such a short time period was difficult, however, participants reported reduced attendance at GP and improved self-management.

\section{P-146 REHABILITATIVE PALLIATIVE CARE - A CHALLENGE ON THE HOSPICE INPATIENT UNIT?}

Sue Cullum. Phyllis Tuckwell Hospice, Farnham, UK

\subsection{6/bmjspcare-2017-hospice.171}

Background Traditionally hospices are seen as providing care and comfort at the end of life. However, many people with a terminal illness have rehabilitation needs - to maintain or improve function, independence and quality of life. This is increasingly the case as benefits of palliative care are being seen as relevant for a widening population with a range of terminal illnesses, and at different stages in their disease.

Rehabilitative palliative care aims to empower patients to achieve realistic goals to optimise function and independence within the limitations of their illness, and is increasingly recognised as an essential part of holistic palliative care. However, this approach can seem confusing and at odds with caring for the dying, and may be misunderstood by patients, families and health professionals. This misunderstanding may reduce opportunities for patients to optimise their final weeks and months, and may be especially challenging on the hospice inpatient unit.

Aims To explore the views and experiences of inpatient unit staff about rehabilitative palliative care, and how these influence models of care and practice.

To identify barriers and practices that are helpful, and to begin a conversation about ways to widen opportunities for a rehabilitative approach.

Methods

- qualitative semi-structured interviews

- 18 inpatient unit staff

- range of health care professions

- interviews taped and transcribed verbatim.

Results Five themes emerged:

- perceptions of hospice and palliative care

- understanding of 'palliative rehabilitation'

- patient psychology

- staff priorities and experiences

- opportunities and barriers.

Conclusions There are many examples of good rehabilitative practice on the inpatient unit, although these are not often named as such. Barriers to this approach may be rooted in perceptions and misunderstandings at public and organisational levels as well as at the bedside. Opportunities for further developing a rehabilitative approach were seen to depend on time and resources, communication and continuity, teamwork, leadership and identifying 'champions'.

\section{P-147 USING ACTION RESEARCH TO EXPLORE HOW A MULTIDICIPLINARY TEAM CAN ENABLE PATIENTS TO LIVE WELL}

Karen Clarke, Katherine Froggatt. Lancaster University, Lancaster, UK

10.1136/bmjspcare-2017-hospice. 172

Background There is increased interest in how hospices can improve patients' independence whilst supporting them living with a life-limiting illness.

Aim To explore how a rehabilitative approach can be integrated into a 15 -bedded hospice inpatient unit using participatory action research.

Methods Two volunteers, one non-clinical and five clinical members of staff participated in a co-operative inquiry group (CIG) whose aim was to bring about a change in hospice practice whilst reflecting on the activities undertaken and how it affected practice. Purposive sampling was used to ensure that nurses and therapists were represented in the CIG to reflect the potentially different perspectives to providing inpatient palliative care.

The CIG and a patient advisory group assessed whether a rehabilitative approach was suitable in this setting using data from the Integrated Palliative Outcome Scale (IPOS) and Minimum Data Set. The CIG then planned what action to take and after each period of activity, assessed the outcome before further action was planned and executed.

Data collection was concurrent, iterative, informed by the activity that took place and used to influence smaller changes as the study progressed. It was also retrospective and included: notes from ten CIG meetings (25 hours), field notes, research diary, organisation documents and data from 16 questionnaires. A thematic analysis approach was adopted.

Results Preliminary analysis showed that a group of staff and volunteers identifying, owning and acting collaboratively (Pascale \& Sternin 2005) can successfully integrate a rehabilitative approach in to an inpatient setting. However: the terminology, rehabilitative palliative care (RPC), can be both a facilitator and barrier to change finding the balance between enabling and caring can be difficult.

RPC challenges traditional role boundaries between healthcare professionals.

Conclusions Conceptually, there is agreement that RPC is desirable but implementation can be challenging. A multidisciplinary team working collaboratively can mitigate and overcome resistance.

\section{P-148 PACED REHABILITATION AND ENABLEMENT PROGRAMME FOR PEOPLE WITH A LIFE-LIMITING CONDITION}

Nicola MacKinnon, Kim Stuart. Willen Hospice, Milton Keynes, UK

10.1136/bmjspcare-2017-hospice. 173

Background In response to evidence in the Hospice UK document 'Rehabilitative palliative care: enabling people to live fully until they die' we designed a service to address three key areas:

- To proactively engage patients in interventions to improve their activity levels

- To reach out to patients in an environment that for many is less daunting than a hospice setting 
- To remove barriers to accessing hospice services.

Aims Empower palliative care patients to maximise their physical activity in order to promote an ongoing active lifestyle and develop resilience in managing the impact of their condition.

Method Paced Rehabilitation and Enablement Programme (PREP) is a community-based service at Willen Hospice funded by a grant from St James's Place Foundation and was developed in partnership with the local council and secondary care. PREP is an $8-12$ week programme delivered within a gym setting by a clinical team consisting of physiotherapy, occupational therapy, nursing and a gym instructor. The weekly two-hour programme includes exercise and education underpinned by an individual goal orientated ethos. The programme utilises a range of outcome measures to identify goals, progression and evaluate the overall impact of the service.

Results Of the eight patients who have so far completed the programme:

- all have achieved their goals

- all have improved their physical performance (Mean '3 metre timed up and go' increase of $5.8 \mathrm{~s}$; Mean 'sit to stands in 1 min' increase of 67\%)

- all are engaged in ongoing physical activity and participating in valued roles of their everyday life (country dancing, fishing and gym memberships)

- three patients want to return as volunteers to support other patients.

Conclusion We believe PREP is an ideal opportunity to empower palliative care patients to stay active for longer and to regain their sense of value and confidence and could be replicated across palliative care settings.

\section{P-149 EFFECTIVENESS OF PALLIATIVE CARE INTERVENTIONS OFFERING SOCIAL SUPPORT - A SYSTEMATIC REVIEW}

Natasha Bradley, Mari Lloyd-Williams, Chris Dowrick. University of Liverpool, Liverpool, UK

\subsection{6/bmjspcare-2017-hospice.174}

Background Social support is a necessary resource for good quality of life, particularly for individuals managing the psychological challenges of living with a life-limiting illness. Qualitative evidence describes social support obtained during palliative care interventions as valuable to the patient and beneficial to their wellbeing. Improving the quantitative evidence base is necessary to develop the application of economic evaluation in palliative care.

Aim(s) This systematic review aims to summarise existing quantitative evidence on the effectiveness (and cost-effectiveness) of interventions offering social support to individuals with life-limiting illness.

Method Research literature was identified through searching of electronic databases, reference picking and hand searches of key journals. Searches returned a total of 6247 unique titles of which sixteen were eligible for inclusion in this review.

Results Identified interventions included group therapies, group multidisciplinary interventions, and palliative day care. Outcome measures and study designs were heterogeneous. There was very limited use of economic evaluation. Statistically significant results were reported in areas including quality of life, mood disturbance, pain experience and symptom control. Benefits were frequently short term or influenced by participant characteristics (age, gender, baseline distress). Methodological limitations included attrition rates, insensitivity of outcome measures, and difficulties in capturing a true baseline.

Conclusions Although benefits in both psychological and physical domains were identified from palliative care interventions that include an element of social support, methodological problems and a scarcity of research has resulted in limited evidence of sustained benefit or cost-effectiveness. Existing evidence suggests that social support interventions may be more beneficial to some groups of patients than others.

\section{P-150 SOCIAL SUPPORT IN PALLIATIVE CARE}

Natasha Bradley, Mari Lloyd-Williams, Chris Dowrick. University of Liverpool, Liverpool, UK

\subsection{6/bmispcare-2017-hospice. 175}

Background A holistic approach to supporting a person with life-limiting illness and their families requires a consideration of their social context, including the availability of social support (defined as the resources gained from relationships with others). Hospices offer social environments, enabling opportunities to interact with others, maintain self-esteem and engage in group activities which are reported to be valuable opportunities to improve wellbeing. There has been limited research into the role of social support in palliative care, and whether the provision of such support has a measurable impact on patient outcomes.

Aims This three-year project will use mixed methods to explore services offered by hospices that facilitate social support, leading to a detailed understanding of social support in palliative care.

Methods A survey, disseminated to all hospices in the UK, will contribute knowledge on the establishment and variety of services that offer social support. Qualitative investigation including observations and interviews with service providers will seek to establish the meaning of social support in life-limiting illness and gain detailed understanding of services. A prospective study of patient reported outcome measures will be used to test the effectiveness (and if possible, cost-effectiveness) of these services.

Results An analysis of the results of the survey and preliminary qualitative findings will be presented.

Conclusions The project will contribute knowledge on the variety and significance of social support services in UK hospices and provide evidence for policy and decision makers on the necessity of social support in the context of life-limiting illness.

\section{P-151 HUMAN RIGHTS IN END OF LIFE CARE: DEVELOPMENT AND IMPLEMENTATION OF A PRACTITIONER'S GUIDE}

Jacqui Graves, Sue Hogston. Sue Ryder, London, UK

\subsection{6/bmjspcare-2017-hospice. 176}

Background There have been many reminders of losing sight of our humanity when delivering health care: the Mid-Staffordshire Enquiry (2013) and Winterbourne View (2012). The Care Quality Commission's new approach and the End of Life Care Education Framework for Scotland, are based on human rights principles. 